\title{
PESQUISA BIBLIOGRÁFICA SOBRE EXEMPLARES DA FLORA E FAUNA NO PARQUE NACIONAL DA SERRA DOS ÓRGÃOS, RJ
}

\author{
Walas Cazassa Vieira ${ }^{1}$ \\ Anne Carulliny Monte ${ }^{2}$ \\ Patrick Cadena Teixeira ${ }^{3}$
}

\section{RESUMO}

A preservação ambiental é de extrema importância para manter o equilíbrio ambiental no planeta. As unidades de conservação (UC) foram criadas no intuito de preservar o meio ambiente em diversas regiões. O Parque Nacional Serra dos Órgãos (PARNASO) é uma UC que abriga grande parte da flora e da fauna da Mata Atlântica brasileira, tornando-o uma importante unidade para a preservação da natureza. O PARNASO está situado no estado do Rio de Janeiro, nos municípios de Teresópolis, Petrópolis, Guapimirim e Magé, tendo uma área aproximada de 20.024 hectares. Apresenta clima mesotérmico brando superúmido com temperatura média anual variando de $13^{\circ}$ a $23^{\circ} \mathrm{C}$, relevo escarpado com grande variação de altitude. No parque nascem diversos rios como: Soberbo, Bananal, Sossego, Inhomirim, Magé, Santo Aleixo, Inconha e Corujas. O parque abriga diversas espécies animais e vegetais e neste artigo estão listadas e caracterizadas 6 espécies do reino Plantae e 6 espécies do reino Animalia são elas: Adenocalymma comosum, Chorisias speciosa, Buddleja stachyoides, Phalaenopsis hybridus, Tillandsia cyane, Euterpe edulis, Nasua nasua, Procyon cancrivorus, Cerdocyon thous, Brachyteles arachnoides, Alouatta guariba, Cebus nigritus.

Palavras-Chave: Preservação; biodiversidade; flora e fauna.

\section{BIBLIOGRAPHIC RESEARCH ON FLORA AND FAUNA EXEMPLARS IN SERRA DOS ORGÃOS NATIONAL PARK, RJ}

\begin{abstract}
The nature preservation is very important for the world's natural balance. The protected areas were created to preserve the nature in many places. The 'Serra dos Órgãos' National Park (PARNASO) protect the flora and fauna of the Atlantic Forest, therefore a very important area. The PARNASO is located in the state of Rio de Janeiro, municipalities of Teresópolis, Petrópolis, Guapimirim and Magé, with 20.024 hectares. The climate is mesothermal with temperature between $13^{\circ}$ to $23^{\circ} \mathrm{C}$, according change of relief and altitude. The Park has the sources of the Soberbo, Bananal, Sossego, Inhomirim, Magé, Santo Aleixo, Inconha e Corujas rivers. Several species are found in PARNASO. In this article are listed and characterized 6 species of the kingdom Plantae and 6 species of the kingdom Animalia. They are: Adenocalymma comosum, Chorisias speciosa, Buddleja stachyoides, Phalaenopsis hybridus, Tillandsia cyane, Euterpe edulis, Nasua nasua, Procyon cancrivorus, Cerdocyon thous, Brachyteles arachnoides, Alouatta guariba, Cebus nigritus.
\end{abstract}

Keywords: Preservation; biodiversity; flora and fauna.

\section{INTRODUÇÃO}

\footnotetext{
${ }^{1}$ Universidade Unigranrio. E-mail: profwalascazassa@yahoo.com.br

2 Universidade Federal do Rio de Janeiro. E-mail: carulmonte@gmail.com

${ }^{3}$ Sociedade de Ensino Sant'Anna e Coutinho: E-mail: patrickcadena2013@gmail.com
} 
O ser humano vem moldando a natureza ao seu redor a fim de facilitar sua própria sobrevivência. $\mathrm{O}$ crescimento constante e não consciente das regiões metropolitanas não leva em conta a preservação dos recursos naturais (JARDIM, 2005), o que pode causar problemas como o aumento na extinção de espécies, a alteração do clima e dos ciclos biogeoquímicos, resultando em um desequilíbrio em todo o planeta (LANDE, 1988). As doenças causadas por infecções virais e parasitárias são diretamente influenciadas pelo desmatamento proveniente da urbanização, extrativismo, atividades agrícolas, mineração e desenvolvimento hidrelétrico (WALSH; MOLYNEUX; BIRLEY, 1993).

Vê-se então a necessidade de uma relação harmoniosa entre o homem e o meio ambiente. Apesar de ter havido um aumento na sensibilização das pessoas em relação à preservação do meio ambiente, o mesmo ainda não é suficiente para conter a degradação ambiental (LANDE, 1988). Em um esforço por parte dos governos, foram criadas as unidades de conservação ambiental. Entende-se como unidade de conservação uma área com características naturais relevantes, delimitada por lei, com objetivos de conservação e proteção (Lei n ${ }^{0} 9.985$ de 18 de julho de 2000). A importância dessas zonas de preservação é evidente em diversas regiões, o que pode ser corroborado através de pesquisas que indicam, por exemplo, que, em áreas do Cerrado brasileiro que estavam protegidas da ação do homem, as características vegetais evoluíram de uma vegetação tipicamente aberta com predomínio de plantas herbáceas para uma vegetação mais fechada, caracterizada pela presença de plantas arbustivas (PINHEIRO \& DURIGAN, 2009).

No Brasil, as unidades de conservação são divididas em duas categorias: de Proteção Integral e de Uso Sustentável. As áreas de Proteção Integral, em geral, são maiores e se destinam a fins educativos, recreativos e pesquisas científicas (MACHADO et al., 2004). Já as áreas de Uso Sustentável permitem diferentes tipos de interferência humana, tendo a conservação em segundo plano (RYLANDS, BRANDON, 2005).

O primeiro Parque Nacional brasileiro surgiu em 1937 e, nas décadas seguintes, outros foram sendo criados. Contudo, somente no ano 2000 as unidades de conservação no Brasil passaram e ser amparadas legalmente por um conjunto mais sistematizado de normas através da Lei $\mathrm{n}^{\circ}$ 9.985, de 18 de julho (RYLANDS; BRANDON, 2005). O Parque Nacional da Serra dos Órgãos tem como função conservar a biodiversidade da área em que o parque está inserido. O parque abrange 20.024 hectares dos municípios de Teresópolis, Petrópolis, Guapimirim e Magé com mais de 3000 espécies (ICMBio, 2007). Ele abriga a maior quantidade de vegetação remanescente da Mata Atlântica (BREVES et al., 2013), bioma que já perdeu mais de $93 \%$ de sua área original. Antes cobrindo grandes extensões, as florestas foram sendo historicamente fragmentadas, na maior parte dos casos em áreas pequenas e separadas entre si (JÚNIOR et al., 2008).

Sobre o clima regional, Oliveira et al (2007) diz:

O clima é mesotérmico brando superúmido com temperatura média anual variando de $13^{\circ}$ a $23^{\circ} \mathrm{C}$, umidade relativa do ar de 80 a $90 \%$ e índice pluviométrico médio de $2.000 \mathrm{~mm}$. Possui verões brandos, sem estação seca, caracterizado pela abundância de precipitações nos meses de outubro a 
março, nos quais concentram cerca de 70 a $80 \%$ das precipitações e inverno com temperaturas e pluviosidade menores, atingindo o mínimo entre os meses de junho e julho.

O relevo é escarpado com uma grande variação de altitude. O nome Serra dos Órgãos deriva do nome do instrumento musical órgão, devido à semelhança das serras do parque com esse instrumento musical (ALVES, 2007). Essa é uma região vulnerável a ocorrência de diferentes tipos de movimentos de massa, tais como: queda de blocos, corridas de massa, escorregamentos translacionais e rotacionais (GUIMARÃES et al., 2009). Esse parque é muito utilizado para a prática do montanhismo e um marco para o montanhismo brasileiro foi a conquista do Dedo de Deus, situado no município de Guapimirim, em abril de 1912 por um grupo de brasileiros (BODENS, 2013)

A área na qual está situado o parque apresenta uma diversidade de solos com, aproximadamente, cinco tipos diferentes: Latossol Amarelo, Latossol Vermelho Amarelo, Latossol Vermelho, Litossol e os solos hidromórficos (ALVES, 2007). A dinâmica das massas de ar nesta região se caracteriza pelo domínio da Massa Tropical Atlântica, contudo a Serra dos Órgãos funciona como uma barreira impedindo a entrada das massas de ar vindas do Atlântico (JÚNIOR et al., 2008). Segundo o ICMBio, Diversos rios apresentam nascente no parque, como os rios Soberbo, Bananal, Sossego, Inhomirim, Magé, Santo Aleixo, Iconha e Corujas, todos desaguando na Bahia de Guanabara.

\section{METODOLOGIA}

Esse trabalho envolve uma pesquisa realizada a partir da revisão de literatura que, segundo Gil (2010), consiste em uma análise de materiais, como livros, artigos, documentos, entre outros, já publicados sobre o tema a ser abordado, com o intuito de analisar diversas perspectivas e buscar o embasamento teórico para a discussão de tal tema. No presente caso, os temas em questão são as unidades de conservação biológica e, particularmente, uma unidade de proteção integral: o Parque Nacional da Serra dos Órgãos, localizado no estado do RJ.

As pesquisas foram realizadas em artigos científicos, em trabalhos do ensino superior dos tipos TCCs, Teses e Dissertações, em artigos publicados em periódicos na internet e no site do ICMBio (Instituto Chico Mendes de Biologia), através de um computador com acesso à internet. Foram consultados materiais abordando os seguintes assuntos: preservação ambiental, degradação natural, unidades de conservação e a importância das áreas de preservação para a flora e a fauna da Mata Atlântica. Foi proposta uma relação entre a degradação ambiental e as unidades de conservação para que através da mesma chegue-se a preservação do meio natural enfatizando, assim, a importância das áreas de preservação para o combate à destruição da natureza através do cuidado da biodiversidade de espécies animais e vegetais.

Juntamente com este artigo, foram criadas doze fichas catalográficas em papel tamanho A4 para exposição de 12 espécies, sendo 6 do Reino Plantae e 6 do Reino Animalia presentes no PARNASO segundo o catálogo de espécies disponibilizado pelo próprio site do 
parque. Essas espécies foram escolhidas arbitrariamente e foi realizada uma pesquisa sobre cada uma. No subtópico "Biologia", trata-se dos aspectos morfofisiológicos da espécie escolhida; em "Ecologia", procura-se expor qual habitat e nicho ecológico essas espécies possuem, além das relações ecológicas que as mesmas podem desenvolver no ambiente. Buscou-se também expor a classificação taxonômica das espécies e as curiosidades relacionadas a tais indivíduos, onde os temas procurados acerca de cada um foram expostos na ficha de forma clara, concisa e de fácil interpretação por qualquer pessoa que tenha interesse em conhecer um pouco sobre uma pequena amostra de espécies do parque, a fim de garantir informações pertinentes em relação aos conteúdos e espécies pesquisados.

\section{RESULTADOS E DISCUSSÃO}

A seguir, serão expostos os resultados obtidos a partir da pesquisa bibliográfica realizada. Como dito anteriormente, foram escolhidas doze espécies presentes em área abrangida pelo parque. As mesmas serão descritas no decorrer da seção.

\section{Espécies Vegetais}

I - Adenocalymma comosum

REINO: Plantae

DIVISÃO: Magnoliophyta

CLASSE: Magnoliopsida

ORDEM: Lamiales

FAMÍLIA: Bignoniaceae

\section{GÊNERO: Adenocalymma}

Essa planta pode ser encontrada no litoral brasileiro em biomas da Mata Atlântica. Possui 2 períodos de floração por ano onde produz inúmeras flores por dia. Hermafrodita e com frutos deiscentes. Pode ser classificada como eudicotiledônea por possuir flores com 5 pétalas, raiz axial, feixes vasculares dispostos ordenadamente e nervuras foliares em formato de rede. Ela necessita de um suporte para se sustentar, entrando assim, na categoria de lianas.

Pode ser utilizada como uma importante planta medicinal para auxiliar no tratamento de inúmeras doenças. Segundo Almeida-Soares (2010), essa espécie pode ser empregada no tratamento do colesterol alto.

II - Chorisia speciosa

REINO: Plantae

DIVISÃO: Magnoliophyta

CLASSE: Magnolipsida

ORDEM: Malvales

FAMÍLIA: Malvaceae 


\section{GÊNERO: Chorisia}

Pode ser encontrada nas florestas mesófilas semidecíduas na região centro-sul do Brasil. Hermafrodita e com frutos deiscentes. Pode ser classificada como eudicotiledônea por possuir raiz axial, feixes vasculares dispostos ordenadamente flores com 5 pétalas e nervuras foliares em formato de rede. É uma espécie de grande porte podendo atingir até $30 \mathrm{~m}$ de altura.

É constantemente utilizada na ornamentação e no paisagismo. Segundo Lorenzi (1992 - p.352), essa planta possui uma forte atuação na economia para a confecção de canoas e forros para móveis.

III - Buddleja stachyoides

REINO: Plantae

DIVISÃO: Magnoliophyta

CLASSE: Magnolipsida

ORDEM: Lamiales

FAMÍLIA: Scrophulariaceae

GÊNERO: Buddleja

Nativa de regiões com clima temperado em países da Ásia, África e Américas. No Brasil ela pode ser encontrada tanto no Cerrado quanto na Mata Atlântica. É uma planta heliófita, pois precisa de intensa exposição ao sol. Ela é uma planta eudicotiledônea, pois apresenta flores com 4 pétalas, feixes vasculares dispostos ordenadamente, raiz axial e nervuras foliares em formato de rede. A planta pode medir cerca de um metro.

Essa planta apresenta grande importância no tratamento medicinal. Segundo Lorenzi (2008), essa planta é capaz de atuar como anti-inflamatório, analgésico, calmante e auxilia no tratamento das hemorroidas.

IV - Phalaenopsis hybridus

REINO: Plantae

DIVISÃO: Magnoliphyta

CLASSE: Liliopsida

ORDEM: Asparagales

FAMÍLIA: Orchidaceae

GÊNERO: Phalaenopsis

A família Orchidaceae é muito difundida em todo o globo com exceção das regiões polares. O gênero Phalaenopsis é proveniente da Austrália e Ásia. Podem ser classificadas como epífita ou rupícolas, que corresponde a ter a sua vida sobre árvores ou sobre pedras respectivamente. São classificadas como monocotiledôneas, pois possuem pétalas brancas em 
um múltiplo de três, raiz fasciculada, feixes vasculares dispostos desordenadamente e nervuras foliares dispostas linearmente.

Essa planta tem um grande valor econômico devido a sua importância para a ornamentação de escritórios e casas. Estimativas dizem que em seis anos, entre 1996 e 2002, as vendas de orquídeas e o valor arrecadado quase triplicaram (WANG, 2003).

V - Tillandsia cyanea

REINO: Plantae

DIVISÃO: Magnoliophyta

CLASSE: Liliopsida

ORDEM: Poales

FAMÍLIA: Bromeliaceae

GÊNERO: Tillandsia

Pode ser encontrada entre o sul dos Estados Unidos e a Argentina. Podem ser epífitas ou rupícolas. Ela pode ser classificada como monocotiledônea por apresentar pétalas trímeras, raiz fasciculada, Feixes vasculares dispostos desordenadamente e nervuras foliares dispostas linearmente. As pétalas podem apresentar uma grande variedade de cores chamativas.

Essa planta é capaz de abrigar um micro ecossistema devido a sua capacidade de acumular água e microrganismos nas flores, conferindo assim a ela uma grande importância ecológica para a natureza (Benzing 2000).

VI - Euterpe edulis

REINO: Plantae

DIVISÃO: Magnoliophyta

CLASSE: Liliopsida

ORDEM: Arecidae

FAMÍLIA: Arecaceae

GÊNERO: Euterpe

Planta típica da Mata Atlântica no litoral brasileiro. Perenifólia, ombrófila, monoica e monocotiledônea. Apresenta flores amarelas com pétalas trímeras, raiz fasciculada, feixes vasculares dispostos desordenadamente, nervuras foliares dispostas linearmente e frutos carnosos. São plantas de grande porte podendo alcançar cerca de $20 \mathrm{~m}$ de altura.

A extração da madeira é muito utilizada na construção. É utilizada para confeccionar ripas, caibros e escoras de andaimes. Além disso, pode ser utilizada para a alimentação e ornamentação (LORENZI, 1992).

A figura 01 mostra exemplares de cada espécie vegetal citada. 
Figura 01: espécies vegetais citadas no texto. Em A: Adenocalymma comosum; B: Chorisia speciosa; C: Phalaenopsis kybridus; D: Tillandsia cyanea; E: Buddleja stachyoides; F: Euterpe edulis

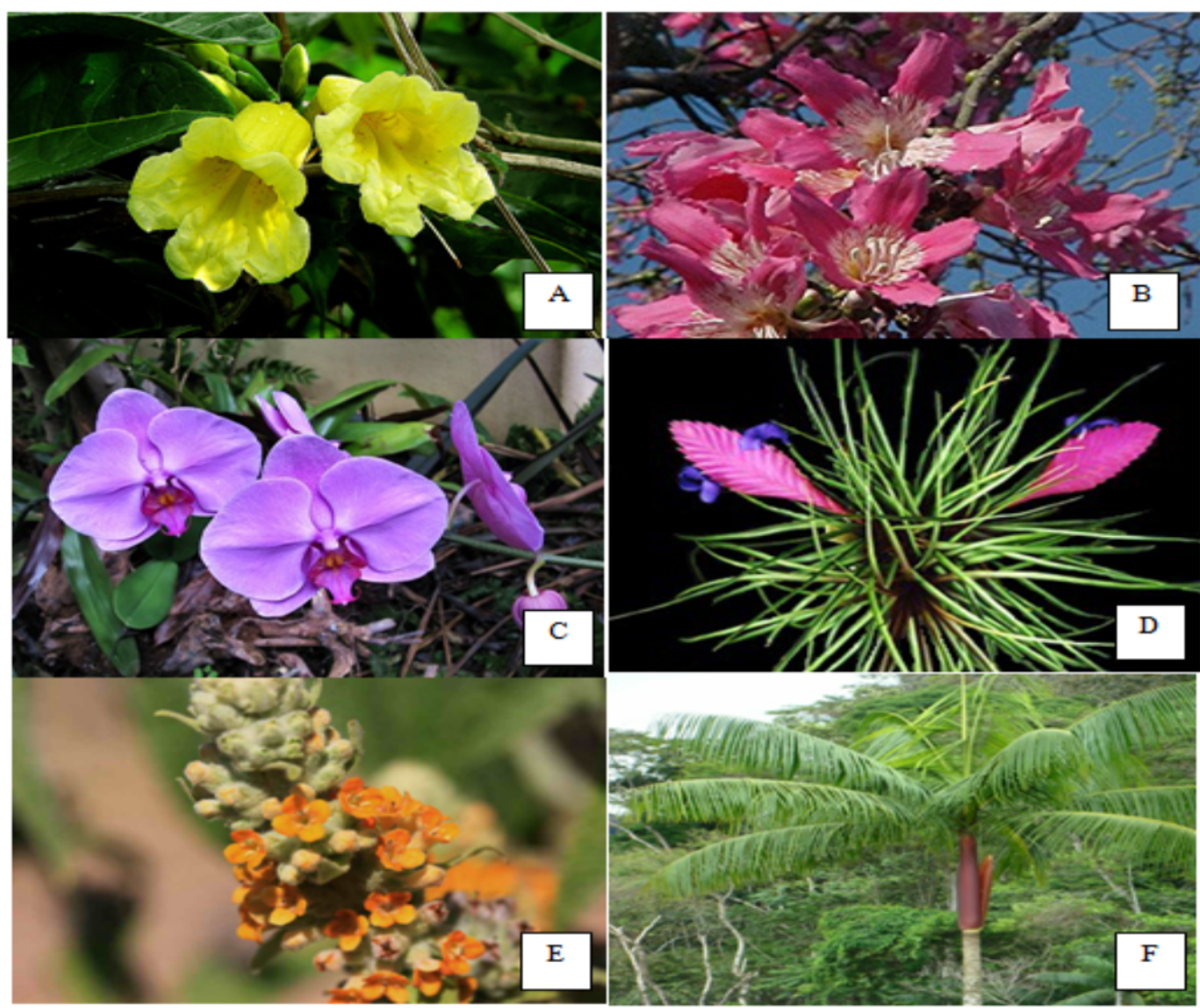

Fontes: Popovkin (2009), Amazon (2017), Gurni (2006), Quintal (2017), Ecoterrazas (2017) e Árvores do Brasil (2017)

Espécies Animais

I - Nasua nasua

REINO: Animalia

FILO: Chordata

CLASSE: Mammalia

ORDEM: Carnivora

FAMÍLIA: Procyonidae

GÊNERO: Nasua 
Encontrado em países da América do Sul, geralmente em florestas de altitudes elevadas. Animais onívoros com dieta composta por pequenos invertebrados e frutas. São animais com hábitos diurnos e que dormem em árvores.

Segundo Gompper (1998), eles apresentam pelagem marrom com o focinho em tons de preto, cauda longa e com pelagem robusta, porém sem preensibilidade, garras longas, orelhas curtas e focinho grande.

II - Procyon cancrivorus

REINO: Animalia

FILO: Chordata

CLASSE: Mammalia

ORDEM: Carnivora

FAMÍLIA: Procyonidae

GÊNERO: Procyon

Podem ser encontrados em países da América do Norte ou América do Sul, geralmente, próximos a praias, lagoas, pântanos ou manguezais. São animais onívoros com dieta baseada em peixe, insetos e frutas. Apresentam hábitos noturnos.

Segundo Lima (2010), eles possuem pelagem em tons de cinza com coloração diferenciada na região dos olhos e focinho. Possui cauda longa, membros posteriores maiores, orelhas curtas e focinho pontudo.

III - Cerdocyon thous

REINO: Animalia

FILO: Chordata

CLASSE: Mammalia

ORDEM: Carnivora

FAMÍLIA: Canidae

GÊNERO: Cerdocyon

Encontrados em vários países da América do Sul. Eles não apresentam dimorfismo sexual e são animais onívoros com dieta baseada em frutas, insetos e outros vertebrados. Frutas são a base da alimentação, insetos e vertebrados raramente servem de alimento para C.Thous.

Segundo Berta (1982), esse animal possui pelagem marrom avermelhada e preta. Apresenta patas robustas, cauda longa com cerca de $30 \mathrm{~cm}$ e focinho pontudo. Os filhotes passam a se alimentar de sólidos a partir de 30 dias de vida.

IV - Brachyteles arachnoides 
REINO: Animalia

FILO: Chordata

CLASSE: Mammalia

ORDEM: Primates

FAMÍLIA: Atelidae

GÊNERO: Brachyteles

Podem ser encontrados no bioma de Mata Atlântica no litoral brasileiro. Sua alimentação é baseada em folhas, sementes e frutas. Possuem hábitos diurnos e atuam como polinizador devido à ingestão de sementes.

Segundo Silva (2004), a coloração da pelagem espessa que varia entre branco, amarelo e marrom. Movimenta-se pela braquiação, devido a isso possuem os braços maiores que as pernas. Possui cauda longa e preênsil.

V - Alouatta guariba

REINO: Animalia

FILO: Chordata

CLASSE: Mammalia

ORDEM: Primates

FAMÍLIA: Atelidae

GÊNERO: Alouatta

Presentes em florestas tropicais da América do Sul. Alimentam-se de folhas e passam grande parte do dia descansando para digerir seus alimentos. Eles apresentam dimorfismo sexual na fase adulta.

Segundo Jardim (2005), possuem coloração marrom-avermelhada, possuindo na região da mandíbula uma espécie de "barba" e não apresentam pelos na face Eles possuem cauda longa e preênsil e um ronco muito forte e característico.

VI - Cebus nigritus

REINO: Animalia

FILO: Chordata

CLASSE: Mammalia

ORDEM: Primates

FAMÍLIA: Cebidae

GÊNERO: Cebus 
Podem ser encontrados na América do Sul e Central. No Brasil, estão distribuídos na Floresta Amazônica, Mata Atlântica, Cerrado e Caatinga. São animais diurnos e possuem dieta baseada em frutos, sementes e folhas.

Segundo Santos (2009), são animais de porte médio e com pelagem preta ou marrom. No topo da cabeça possuem uma pelagem semelhante a um topete. A sua cauda é longa e semipreênsil e possui grande capacidade de manipular objetos.

A figura 02 mostra as espécies animais citadas no texto.

Figura 02: animais citados no texto. Em A: Nasua nasua; B: Procyon cancrivorus;C:

Cerdocyon thous; D: Brachyteles arachnoides; E: Alouatta guariba e F: Cebus nigritus
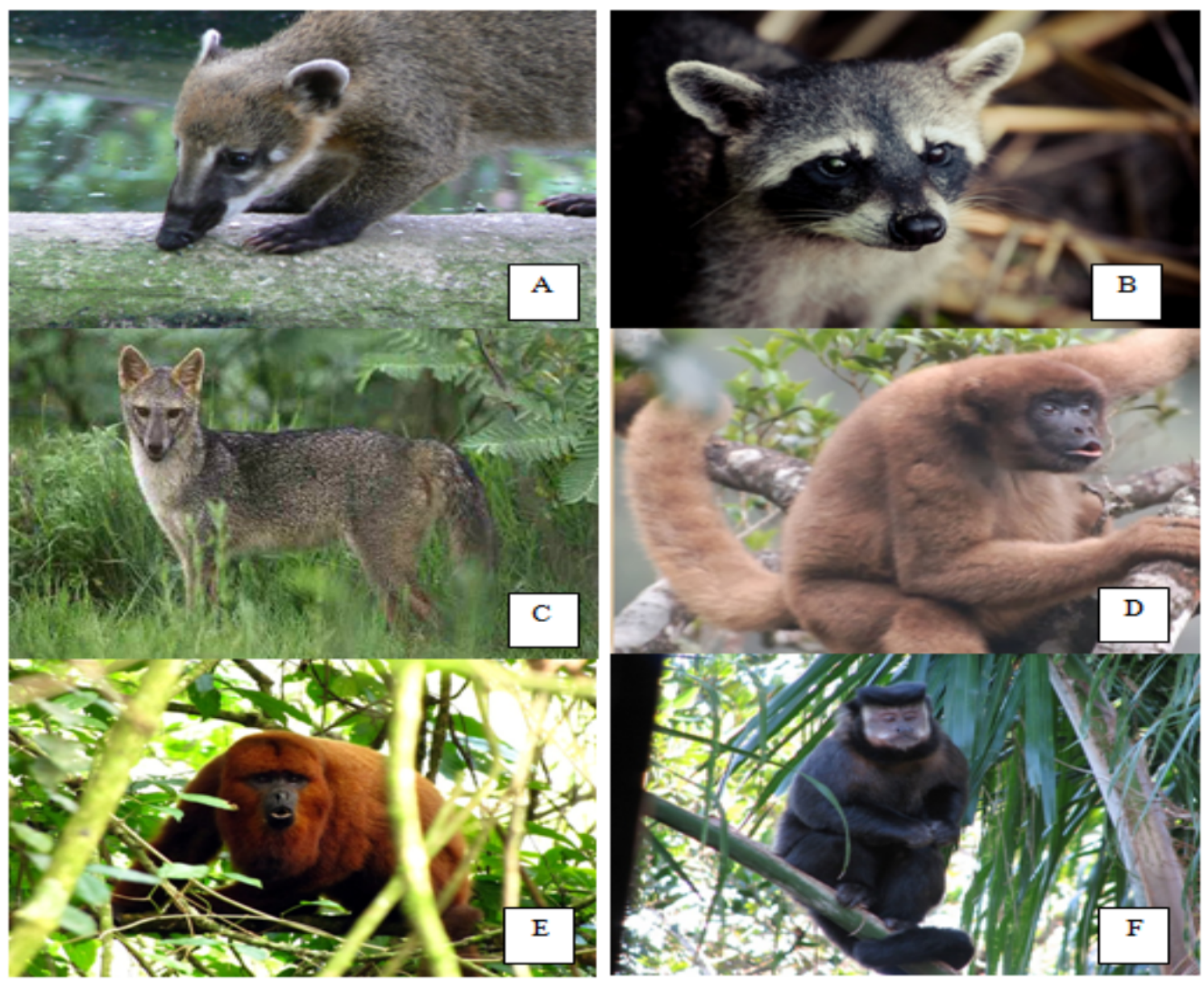

Fontes: Wikimedia (2009), Beaubois (2016), Taipan (2014), Ui7.co (2017), Kindel (2016) e ICMBio (2017)

No quadro I, a seguir, uma síntese das espécies e suas informações básicas.

Quadro I: Síntese de informações sobre as espécies citadas no texto

\begin{tabular}{|l|l|l|l|}
\hline NOME & REINO & REGIÃO & IMPORTÂNCIA \\
\hline
\end{tabular}




\begin{tabular}{|c|c|c|c|}
\hline $\begin{array}{l}\text { Adenocalymma } \\
\text { comosum }\end{array}$ & Plantae & Litoral brasileiro & Medicinal \\
\hline Chorisias speciosa & Plantae & $\begin{array}{c}\text { Centro-Sul do } \\
\text { Brasil }\end{array}$ & $\begin{array}{c}\text { Ornamentação e } \\
\text { extração }\end{array}$ \\
\hline $\begin{array}{c}\text { Buddleja } \\
\text { stachyoides }\end{array}$ & Plantae & Hemisfério Sul & Medicinal \\
\hline $\begin{array}{c}\text { Phalaenopsis } \\
\text { hybridus }\end{array}$ & Plantae & Austrália e Ásia & Ornamentação \\
\hline Tillandsia cyane & Plantae & Américas & Ecológica \\
\hline Euterpe edulis & Plantae & Litoral Brasileiro & $\begin{array}{c}\text { Ornamentação e } \\
\text { extração }\end{array}$ \\
\hline NOME & REINO & REGIÃO & PELAGEM \\
\hline Nasua nasua & Animalia & América do Sul & Marrom \\
\hline $\begin{array}{c}\text { Procyon } \\
\text { cancrivorus }\end{array}$ & Animalia & América do Sul & Cinza \\
\hline Cerdocyon thous & Animalia & América do Sul & Marrom/Preta \\
\hline $\begin{array}{l}\text { Brachyteles } \\
\text { arachnoides }\end{array}$ & Animalia & Litoral Brasileiro & Branca/Amarela \\
\hline Alouatta guariba & Animalia & América do Sul & $\begin{array}{c}\text { Marrom- } \\
\text { avermelhada }\end{array}$ \\
\hline Cebus nigritus & Animalia & $\begin{array}{c}\text { América do Sul e } \\
\text { Central }\end{array}$ & Preta \\
\hline
\end{tabular}

Fonte: Dados da pesquisa

\section{CONCLUSÃO}

A composição desse trabalho pôde confirmar a importância das unidades de conservação para a flora e a fauna brasileira. Análise feita a respeito das 12 espécies selecionadas mostrou a importância da preservação das mesmas e de seus habitat. Foram observadas espécies que influenciavam diretamente na vida humana, e outras espécies que eram peças chaves para a manutenção do meio ambiente.

Esse artigo é importante para incentivar a conscientização e a valorização das unidades de conservação e do meio ambiente. O bioma de Mata Atlântica, no qual está situado o parque, é extremamente depredado por ações antrópicas, então é de extrema 
importância a existência dessa unidade de conservação a fim de proteger e preservar a natureza ali presente.

\section{REFERÊNCIAS BIBLIOGRÁFICAS}

ALMEIDA-SOARES, Stela; POLATTO, Leandro P.; DUTRA, João C. S.; TOREZANSILINGARDI, Helena M. Pollination of Adenocalymma bracteatum (Bignoniaceae): floral biology and visitors. Neotropical Entomology, v. 39, n. 6, p.941-948, dez. 2010. Springer Nature.

ALVES, Gilberto Terra Ribeiro. Aspectos da história de vida de Guadua tagoara (Nees) Kunth (Poaceae: Bambuseae) na Serra do Órgãos, RJ. 2007. 113 f. Dissertação (Mestrado) - Curso de Ecologia e Recursos Naturais, Ppgern, Universidade Federal de São Carlos, São Paulo, 2007.

BENZING, D. H. 2000. Bromeliaceae: profile of an adaptive radiation. Cambridge University, Cambridge, England

BERTA, A. Cerdocyon thous. Mammalian Species, Lawrence, v.186, p. 1-4, 1982.

BODENS, Johannes. Diretrizes para avaliação do impacto ambiental da prática de boulder em Parques Nacionais e Estaduais: $O$ caso do Parque Estadual dos Pirineus (GO). 2013. 78 f. TCC (Graduação) - Curso de Geografia, Departamento de Geografia, Universidade de Brasília Departamento de Geografia, Brasília, 2013.

BREVES, Paula; STRAVS Austem; DIAS Andrade; PISSINATTI Alcides; BOUBLI, Jean Philippe. Uso do Chão por Brachyteles arachnoides no Parque Nacional Serra Dos Órgãos, Teresópolis, Brasil. Neotropical Primates, [s.1.], v. 20, n. 1, p.52-54, jun. 2013. Conservation International.

GOMPPER, Matthew E.; DECKER, Denise M. Nasua nasua. Mammalian Species, [s.1.], n. 580, p.1-9, 1 jun. 1998. Oxford University Press (OUP)

GUIMARÃES, Renato Fonte; GOMES, Roberto Arnaldo Trancoso; JÚNIOR, Osmar Abílio de Carvalho; MARTINS, Éder de Souza; OLIVEIRA, Sandro Nunes de; FERNANDES, Nelson Ferreira. Análise temporal das áreas susceptíveis a escorregamentos rasos no Parque Nacional da Serra dos Órgãos (RJ) a partir de dados pluviométricos. Revista Brasileira de Geociências, v. 39, n. 1, p.190-198, mar. 2009.

JARDIM, Márcia Maria de Assis. Ecologia Populacional de Bugios-Ruivos (Alouatta guariba) nos Municípios de Porto Alegre e Viamão, RS, Brasil. 2005. $124 \mathrm{f}$. Tese (Doutorado) - Curso de Ecologia, Instituto de Biologia, Universidade Estadual de Campinas, Campinas, 2005.

JÚNIOR, Osmar Abílio de Carvalho; COELHO, Marcus Alberto Nadruz; MARTINS, Éder de Souza; GOMES, Roberto Arnaldo Trancoso; JÚNIOR, Felipe Couto; OLIVEIRA, Sandro Nunes de; SANTANA, Otacílio Antunes. Mapeamento da vegetação na floresta atlântica 
usando o classificador de árvore de decisão para integrar dados de sensoriamento remoto e modelo digital de terreno. Revista Brasileira de Geofísica, São Paulo, v. 26, n. 3, set. 2008.

LANDE, Russel. Genetics and Demography in Biological Conservation. Science, New Series, v. 241, n. 4872, p.1445-1460, 1988.

LIMA, Vanessa Morais; PEREIRA, Firmino Cardoso; PEREIRA, Kleber Fernando. STUDO MORFOLÓGICO DOS MÚSCULOS DO ANTEBRAÇO DE MÃOPELADA, Procyon cancrivorus CUVIER, 1798. Bioscience Journal, Uberlândia, v. 26, n. 1, p.109-114, fev. 2010 .

LORENZI, H. Árvores brasileiras: manual de identificação e cultivo de plantas arbóreas nativas do Brasil. Nova Odessa: Plantarum, p.352, 1992.

LORENZI, H.; MATOS, F. J. A. Plantas medicinais no Brasil: nativa e exóticas. 2 ed. São Paulo: Instituto Plantarum de Estudos da Flora Ltda, 2008. p. 487.

OLIVEIRA, Sandro Nunes de; JÚNIOR, Osmar Abílio de Carvalho; MARTINS, Éder de Souza; SILVA, Telma Mendes da; GOMES, Roberto Arnaldo Trancoso, GUIMARÃES, Renato Fontes. Identificação de Unidades de Paisagem e sua Implicação para o Ecoturismo no Parque Nacional da Serra dos Órgãos, Rio De Janeiro. Revista Brasileira de Geomorfologia, v. 8 , n. 1, p.87-107, 17 nov. 2007.

PINHEIRO, Eduardo da Silva; DURIGAN, Giselda. Dinâmica espaço-temporal (1962-2006) das fitofisionomias em unidade de conservação do Cerrado no sudeste do Brasil. Revista Brasil, v. 32, n. 3, p.441-454, set. 2009.

RYLANDS, Anthony B.; BRANDON, Katrina. Unidades de conservação brasileiras. Megadiversidade, v. 1, n. 1, jul. 2005.

SANTOS, L. B.; REIS, N. R. dos. Estudo comportamental de Cebus nigritus (Goldfuss, 1809) (Primates, Cebidae) em cativeiro. Semina: Ciências Biológicas e da Saúde, Londrina, v. 30, n. 2, p.175-184, mar. 2009

SILVA, Cristina Isis Buck. Utilização de padrões de locomoção por um grupo de muriquis Brachyteles arachnoides (E.Geofroy,1806) em um fragmento de Floresta Atlântica no Município de Castro, PR. 2004. 24 f. Monografia (Especialização) - Curso de Ciências Biológicas, Universidade Federal do Paraná, Curitiba, 2004.

WALSH, J., MOLYNEUX, D. \& BIRLEY, M. (1993). Deforestation: Effects on vector-borne disease. Parasitology, 106 (S1), S55-S75.

WANG, Y.T. (2003) Learn how to grow Phalaenopsis orchids. GMPRO, 40-43. 